\title{
107
}

Reprinted from the Journal of Chemical Physics, Vol. 32, No. 2, 617-618, February, 1960 Printed in U. S. A.

\section{Radiant Energy Emission from Excited Harmonic Oscillators*}

\author{
S. S. Penner \\ Daniel and Florence Guggenheim Jet Propulsion Center, \\ California Institute of Technology, \\ Pasadena, California
}

(Received October 14, 1959)

$\mathbf{T}$ WE total radiant energy emitted by spontaneous transitions ${ }^{1}$ is independent, to the harmonic oscillator approximation, of the initially excited vibrational level(s) for a fixed total energy input. The preceding statement may be verified readily by the following considerations.

Let $x_{n}(t)$ denote the number of vibrationally excited emitters in the $n$th quantum state at time $t$. Let $n^{*}$ denote the vibrational quantum number of the energy level which is excited initially. Then

$$
d x_{n} * d l=-A_{n *, n *-1} x_{n} *=-A_{1,0} n^{*} x_{n} *
$$

where we use the relation $A_{n, n-1}=n A_{1,0}$ between the Einstein coefficients for spontaneous emission, which is applicable to the harmonic oscillator approximation. It is apparent from Eq. (1) that $x_{n}=N \exp \left(-A_{1,0} n^{*} t\right)$ if $x_{n} *(0)=N$. Similarly,

$$
d x_{n} *-1 / d t=\left[n^{*} N \exp \left(-A_{1,0} n^{*} t\right)-\left(n^{*}-1\right) x_{n} *-1\right] A_{1, \mathrm{e}}
$$

or

$$
\begin{gathered}
x_{n *-1}(t)=n^{*} N \exp \left[-A_{1,0}\left(n^{*}-1\right) t\right]\left[1-\exp \left(-A_{1,0} t\right)\right] ; \\
x_{n-m}(t)=(1 / m !)\left[n^{*} ! /\left(n^{*}-m\right) !\right] N \\
\cdot \exp \left[-A_{1,0}\left(n^{*}-m\right) t\right]\left[1-\exp \left(-A_{1,0} t\right)\right]^{m}, \\
m=0,1, \cdots, n^{*}-1, n^{*} .
\end{gathered}
$$

The set of relations represented by Eq. (2) constitutes a general solution for the number of emitters in any energy level between the ground level $\left(m=n^{*}, n^{*}-\right.$ $m=0)$ and the most highly excited level $(m=0$, $\left.n^{*}-m=n^{*}\right)$.

The total radiant energy transfer equals the product of the number of transitions per second and the energy per transition. To the order of approximation used in the present analysis, $\nu_{n, n-1}=\nu=$ constant. Hence the total radiant energy emitted in unit time is

$$
d \epsilon / d t=A_{1,0} h \nu \sum_{m=n *-1}^{0}\left(n^{*}-m\right) x_{n} *-m(t)
$$

or, using Eq. (2),

$$
d \epsilon / d t=A_{1,0} h \nu n^{*} N \exp \left(-A_{1,0} t\right) .
$$

Reference to Eq. (3) shows immediately that the rate of radiant energy emission is independent of the value of $n^{*}$ for the fixed energy input $n^{*} N h \nu$.

The preceding result permits ready generalization to an arbitrary initial distribution in which $x_{n}(0)=$ $N_{n}$. These distributions relax independently in such a way that the total rate of energy output becomes

$$
\begin{aligned}
d \epsilon_{T} / d t=A_{1,0} h \nu\left(\sum_{n=1}^{\infty} n N_{n}\right) & \exp \left(-A_{1,0} t\right) \\
& =A_{1,0} h \nu N_{T} \exp \left(-A_{1,0} t\right)
\end{aligned}
$$

where

$$
N_{T}=\sum_{n=1}^{\infty} n N_{n}
$$

represents the total number of quanta of energy $h \nu$ distributed among the various vibrational energy states.

* Supported by the Office of Scientific Research, U.S. Air Force, under Contract AF 18(603)-2.

${ }^{1}$ For a general discussion of de-excitation of harmonic oscillators, see K. E. Shuler et al., J. Chem. Phys. 26, 454 (1957); J. Phys. Chem. 61, 849 (1957); J. Chem. Phys. 28, 4 (1958); 29, $366(1958)$. 